\title{
A Comparison of Your Better Life Index and Its Antecedents Across Two Chinese Cultures
}

Jennifer H. Gao, Macao Polytechnic Institute, China

\begin{abstract}
OECD identified 11 topics in Your Better Life Index (YBLI). YBLI is generally accepted as an essential indicator of material living conditions and quality of life. This study compared YBLI in two predominantly Chinese regions (i.e., Macau [a former Portuguese territory in China] and Zhuhai in the Greater Bay Area in the southern part of the People's Republic of China). Data were collected from 446 (231 Macau and 215 Zhuhai) ethnic Chinese residents in the two regions. Results revealed the Macau residents to be much more satisfied with life (significantly higher ratings on more YBLI topics) than their Zhuhai counterparts. Regression analyses revealed Chinese values and family emotional support to be strong predictors of YBLI in Zhuhai, while future prospects and self-efficacy explained most variance for YBLI in Macau. The results of the comparisons are discussed in terms of seminaries and differences in the cultures and economic development of the regions.
\end{abstract}

\section{KEYWORDS}

Chinese Values, Culture, Family Emotional Support, Future Prospects, Self-Efficacy, YBLI

\section{INTRODUCTION}

In the past few decades, China has gained a great deal of interest among international researchers because of its steadily increasing importance to the world economy. Many theoretical and empirical papers have been published since 1978 (the beginning of China's "Open Door" policy) on various aspects of business in China. Employees' job satisfaction, job security and organizational citizenship behavior are a few examples of frequently research variables. However, limited studies of social wellbeing and quality of life in China have been published in the same period. This lack of research tends to imply that there is a clear need to better understand the work-life balance and life satisfaction of the Chinese people. One of the popular ideas regarding the Chinese people is that they are hard-working and easily contented. These characteristics can be tied to Confucian ethics as well as economic growth in nations dominated by Chinese culture (Minkov \& Hofstede, 2011). This research, therefore, is designed to obtain Chinese peoples' attitude towards YBLI by examining each of the 11 topics and factors that might contribute to this important social variable. The findings may shed light on how policymakers should help residents cope with economic uncertainties, and enhance their life satisfaction. 
The need to better understand the Chinese is reinforced in absolute terms by the 1.4 billion Chinese people who live in China, and the number of Chinese people around the world who compose increasing portions of the world's population. Given this large number, the question becomes one of whether attitudes and behaviors differ among Chinese residents who live and work in different regions. On one hand, given the strong arguments in support of the idea that Chinese people maintain their cultural values wherever they go (Lin \& Zhao, 2016), it could be argued that life attitudes that are culturally based would not differ significantly among Chinese residents in different regions. Alternately, to the extent that people's views toward life can be influenced by the prevailing culture in which they live, it could be surmised that differences in life attitudes and behaviors do emerge.

Regarding cultural differences, Hofstede (2015) argued that culture, work-related values and life attitudes are tied together. While concepts such as fame and gain may explain motivation in western cultures, many researchers believe that prevailing values (e.g., thrift and moderation) in China differ from those in western cultures (Jin, Yu \& Kang, 2013).

Instrumental values (e.g., pay, benefits, and working conditions) were often believed to be of low importance in China, however, more recent research suggests the opposite, i.e., that Chinese people do give high importance to such matters as high earnings and pleasant working conditions, and to other extrinsic motivation factors (Nazir et al. 2016).

This study, therefore, focused on the life attitudes of Chinese ethnicity lived in two locations in China (i.e., Macau and Zhuhai) that have been separated by a national border for over 440 years. Macau was a Portuguese possession, and was returned to the Peoples Republic of China (PRC) in 1999. Today, Macau is a "Special Administrative Region" while Zhuhai is a "Special Economic Zone," and the border separating the two is still used. Although they are usually called cities, the term "region" will be used in this paper to reflect the official designation that the PRC government has given to these locations.

\section{THEORETICAL BACKGROUND \& LITERATURE REVIEW}

In this paper, YBLI is examined in relation to self-efficacy and family emotional support, factors which are assumed to be related to people's confidence in themselves and mental health. It is also examined with future prospects, an organizational variable relates to career advancement. Traditional Chinese values (e.g. harmony, respect tradition, reciprocity, etc.), an important social factor is studied to reveal if beliefs and values relate to Chinese people's well being and quality of life.

\section{YBLI}

OECD (2018) considers YBLI to be a more important indicator than GDP. In the words of OECD: there is more to life than the cold numbers of GDP and economic statistics. The 11- topic indicator identified by OECD represents material living conditions and quality of life, and allows comparison of well being across countries. A thorough evaluation system has been developed by OECD to examine and improve happiness level of cities, regions, and countries. Many countries, such as the USA, UK, Russia, Germany, France, Japan, Korea and Australia have adopted the evaluation system to involve citizens to actively engage in policy-making process related to daily lives. The users of YBLI have been encouraged to share their own indexes by adjusting the proportion of different dimensions. Since 2011, OECD has received more than 100,000 results from 180 countries and territories. As everyone can create his or her own index, the results vary from person to person. The responses, which updates daily, have been grouped by country, age and gender, and displayed on the interactive map.

The 11 topics include housing, education, life satisfaction, income, environment, safety, jobs, civic engagement, work-life balance, community and health (OECD, 2018). 


\section{Housing}

Housing is one of people's basic needs. It is not only a shelter built by walls and roofs, it refers to a private place where people could have a rest and feel safe. Housing costs, including rent, gas, electricity, water, and furniture, account for a large proportion of the household budget and represent the largest single expenditure for many families. "In the OECD, households on average spend around $21 \%$ of their gross adjusted disposable income on keeping a roof over their heads. The level of housing costs in household budgets varies from $26 \%$ in New Zealand to $15 \%$ in Korea. In Latvia and the Russian Federation, spending on housing also accounts for $23 \%$ and $19 \%$ of the household budget, respectively" (OECD, 2018).

\section{Education}

Education provides individuals with knowledge, skills and capabilities which are needed for the society and economy. What's more, education may contribute to the quality of life in terms of health and happiness. Studies have shown that people who are well educated live longer and contribute more to the society (Sasson, 2016). According to researches in different regions, people are expected to go through 17 years of education. The duration may extend in some developed country where people are expected to have 21 years of school education (OECD, 2018).

\section{Life Satisfaction}

Life satisfaction measures how people evaluate their life as a whole rather than their current feelings. Measuring feelings can be very subjective, but is nonetheless a useful complement to objective data when comparing quality of life across countries. Subjective data can provide personal evaluations which are often different in different countries. Life satisfaction includes health, education, income and social situation (OECD, 2018).

\section{Income}

Although money cannot buy happiness directly, it is important to increase living standards and create greater well-being. More economic wealth also improves access to qualified education, health care and housing. Financial resources can avoid economic hardship and vulnerability. Living standards have improved a lot in the past few decades, however, there are still many people living in poor condition. Furthermore, the gap of wealth is increasing. According to OECD (2018), the average net-adjusted disposable income of the top $20 \%$ of the population has family income summary as USD64,103 a year, whereas the bottom $20 \%$ live on an estimated USD10,045 a year.

\section{Environment}

Environment condition has an influence on people's health and well-being. Unpolluted environment can be a source of satisfaction, as it improves mental well-being when people could relax themselves in a good living environment. Scientists predicted that air pollution would be the top environmental cause of premature mortality by 2050 (Lelieveld et al., 2015). Although $81 \%$ of people in OECD countries say that they are satisfied with water quality, both air and water pollution have become serious problems in many countries. Greater efforts from government need to be made on environmental management. Protection of resources, environmental protection, and ecological conservation are solid basis for sustainable development.

\section{Safety}

Personal safety refers to the degree people are attached physically, mentally or financially. Crime leads to physical pain and mental anxiety, and the biggest impact on people's well-being is the feeling of vulnerability it causes. Homicide rate is believed to reflect the safety level of an area. According to the latest OECD data, the average homicide rate in the OECD is 3.6 murders per 100,000 inhabitants. 
Domestic violence is also believed to be negatively related with personal safety. In addition, the feeling of safe when people walk alone at night in the area is another important factor to constitute personal safety.

\section{Jobs}

Jobs have a strong relation with happiness. Much domestic violence is due to bad feelings parents get in their company. Jobs determine the family income and consumption level. It includes job security, personal earnings, long-term unemployment rate and employment rate. In the whole world, female employment rate has been greatly raised, but discrimination is still apparent in many countries. The rate of female employment is significantly lower than that in the USA (Deresky, 2017).

\section{Civic Engagement}

Trust in government is a basic element for social stability and well-being. Citizens demand greater transparency on political decisions. Tansparency helps government get more supporters and reduce citizens' anxiety. Voter turnout is a measurement of citizens' participation in political activities. Normally, voter turnout averaged 69\% in OECD countries (OECD, 2018).

\section{Work-Life Balance}

Workers need time to relax themselves to be more productive. Free time in life is an important part of happiness. Balance between work and life takes time to develop smoothly. It is regarded that the government should make sure that workers have time for leisure and personal care.

\section{Community}

Quality of relationship between people is crucial in determining well-being. People are often more enthusiastic when they get along well with their friends. A strong social network can provide emotional support during both good and bad times, whereas a weak social network declines business development opportunities and happiness.

\section{Health}

Good health brings many benefits to people. It includes life expectancy and self-report health. Life expectancy focuses on the length of people's life, and is the most widely used measure of health. Studies (Vaupel, Zhang \& van Raalte, 2011) show that on average, life expectancy is around 80 years, a gain of more than 10 years since 1960 . Women and people who have better life style live longer. Most OECD countries carry out self-administered health servey regularly. The results have been found to be a good predictor of people's future health care.

\section{Self-Efficacy and YBLI}

Self-efficacy is individuals' assessment of their capabilities to organize and to execute actions required to achieve successful levels of performance (Tariq, 2012). It makes a significant difference in how people feel, think and act. Researches indicate that a low sense of self efficacy is linked with psychopathologies like depression and anxiety, while people with high self-efficacy tend to handle difficulties effectively and persist despite failures (Kwok, Cheng \& Wong, 2015). High levels of selfefficacy have also been linked to high levels of innovation, skill in bringing about positive change (Kao, 2017), carrer achievement (Ali \& Menke, 2014), and job satisfaction (Peng \& Mao, 2015).

Perceived self-efficacy is people's beliefs in their capabilities to exercise control over their own functioning and over events that affect their lives. It is one of the most influential traits that make up the core self-evaluation, a higher order construct that represents personal feeling of worthiness, competence and capabilities at work (Kwok, Cheng \& Wong, 2015). Research shows that perceived self-efficacy is related to career optimism (Garcia et al., 2015) and career commitment (Jiang, 2016). 
These results are profounding. As for most people, a job is an essential part of everyday life. The study result by Jiang et al. (2017) revealed that self-efficacy is positively related to overall life satisfaction. Therefore, the following hypothesis is set:

H(1): Self-efficacy has a significant and positive effect on YBLI in Macau and Zhuhai.

\section{Family Emotional Support and YBLI}

Every individual encounters difficulties and challenges during his or her life, and cannot always depend on oneself to solve all problems. Social support is the perception and actuality that one is cared for, has assistance available from other people, and most popularly, that one is part of a supportive social network. Humans are social animals, who offer help to and get support from others. These supportive resources can be emotional, tangible, informational, or companionship and intangible. Family often acts as the primary source of social support, such as emotional sustenance and instrumental assistance (Kwok, Cheng \& Wong, 2015). The sensitive and understanding approach of emotional support encourages communication and reduces stress and fears (Levens, Elrahal \& Sagui, 2016). Emotional support from family is associated with lower psychological distress (Horwitz, Reynolds \& Charles, 2015). It makes people feel comfortable and refreshed, and generates energy to overcome difficulties.

According to Coverdale and Long (2015), family social support shows a substantial and positive association with concurrent happiness. In the findings of Botha and Booysen (2014), supportive intra-family relationships contribute to the level of individual well-being. Furthermore, comparing to those live in extremely or moderately dysfunctional families, individuals live in balanced family functioning are more satisfied with life. Therefore, the following hypothesis can be tendered:

H(2): Family emotional support has a significant and positive effect on YBLI in Macau and Zhuhai.

\section{Future Prospects and YBLI}

Future prospects refer to the opportunities for advancement, bonuses, and other rewards that organizations use in their normal operations. Conceptually, rewards, such as job benefits and opportunities for promotion, increase job satisfaction (e.g., Pandey \& Asthama, 2017) as well as job performance (Schleicher, Greguras \& Watt, 2004). Porter, Riesenmy and Fields (2016) suggested that organizations use promotions and monetary bonuses to motivate employees, thus helping them to grow professionally, which should increase productivity. Further, if employees perceive that their organization provides a rewarding future, employees could become more committed (Cicekli \& Kabasakal, 2017). Such favorable perceptions should also lead to a higher level of life satisfaction. Consequently, it can be expected that:

H(3): Future prospects have a significant and positive effect on YBLI in Macau and Zhuhai.

\section{Chinese Values and YBLI}

Values are referred to as beliefs that are experienced by individuals as standards that instruct how they should function. Values have congnitive, affective, and behavioral dimenstions, and develop through the influence of culture, society and personality, so that individuals can meet their needs in a socially accepted manner (Senge, 2013).

In 1980, Hofstede published a scholarly description of his empirical search for the value dimensions across which cultures vary. The Chinese people are belived to have a unique Chinese value system, a heritage from Confucius that respects reciprocation, harmony, thrift and moderation. These values have been shown to influence attitudes toward loyalty, sharing versus competing, and being contended. Specifically, Confucius elaborated five hierarchical relationships that described 
appropriate codes of conduct. The first was "Righteousness between ruler and subject," whereby fealty and service were to be given to the ruler/leader by the subject/follower and was to be returned by the more-powerful ruler with protection and sustenance for the less-powerful subject. This portrays a paternalistic relationship of benevolent leaders and respectful, obedient servants. The other four relationships (i.e., father-son, husband-wife, older brother-younger brother, and among friends) were prescribed to follow the same model of social behavior, in which dedication from subordinates is expected throughout society.

Research (Ungerer \& Strasheim, 2011) supports that cultural orientation and value systems affect one's behavior and life attitude. Considering the content of the Chinese traditional values, the following hypothesis can be proposed:

H(4): Chinese values have a significant and positive effect on YBLI in Macau and Zhuhai.

\section{Differences Between Two Chinese Cultures}

An objective of this study is to examine whether YBLI will differ across different regions in the same country. To the extent that people from different cultures have different attitudes and values, it would seem logical to expect these differences to include their views well-being and quality of life.

The two Chinese regions of Macau and Zhuhai share a border, but have different cultures. Macau was discovered by the Portuguese and founded as a territory in 1557. As the only European possession in China, Portuguese Macau enjoyed a trading monopoly for the first 300 years until Britain occupied Hong Kong in 1841, after which Macau's status changed to being primarily a resort town for European and Cantonese traders. Macau was returned to Chinese rule in 1999, now has a population of 630,000 people, and became a "Special Administrative Region" (similar to Hong Kong) under the formula of "one country, two systems". Of the two economic systems in Macau and Zhuhai, the one in Macau is strongly capitalistic since gambling/tourism has long been its principal industry, and Macau is the only place in all of China where casinos are legal. The other economic system, namely, the one in Zhuhai, is socialist as it has always been a part of the PRC.

Zhuhai is located in the Greater Bay Area in the southern part of Mainland China and borders Macau on the north and west, and (like Macau) is a geographical neighbor of Hong Kong, which lies to the east across the Pearl River Delta on the South China Sea. Zhuhai was a small fishing village until 1980, when it was designated as one of the first "Special Economic Zones" under Deng Xiaoping's “Open Door Policy.” Zhuhai now has 1.89 million people and, according to the Zhuhai Municipal Government (2018), has become one of the most rapidly developing regions in China.

The majority of residents in both regions are of Chinese ethnicity. Among the majority Chinese, they speak the same Cantonese dialect and share the same Cantonese customs and traditions. However, as previously noted, the economic systems remain different even after the handover of Macau to the PRC (in 1999).

If the Macau Chinese have become westernized during the 440 years that Macau was under Portuguese control, then it could be surmised that the Macau Chinese employees might have been influenced by the Portuguese culture, which for centuries regarded Macau as a tourist resort. Presently (see Direcção dos Serviços de Estatistica e Censos, 2019), about 1/3 of the working population in Macau is employed in the tourist industry, i.e., casinos, hotels, restaurants, recreation, etc. As such, the general lifestyle and work climate tends to be rather relaxed and casual, as opposed to competitive.

On the other hand, the Zhuhai Chinese might be more influenced by the deeply-rooted Confucian values, and they are also more stressed due to fierce competition and pressures of daily life. This would lead to the following hypothesis:

H(5): YBLI in Macau is higher than that in Zhuhai. 


\section{METHOD}

\section{Respondents}

Data were collected from 446 residents (201 males and 245 females) in Macau $(\mathrm{n}=231)$ and Zhuhai $(n=215)$. All respondents were ethnic Chinese, aged from 18 to 56 years $(M=26.55, S D=6.08)$. Most respondents $(73.60 \%)$ had completed their secondary school education, with the remainder (26.40\%) having completed at least a tertiary education. The respondents had spent an average of 3.30 years $(\mathrm{SD}=2.97)$ in their current jobs. As for industry, $50.10 \%$ of the respondents were employed in manufacturing or construction, $28.60 \%$ in services, $19.10 \%$ in sales, and $2.20 \%$ in an unspecified "other" industry. Most respondents (62.50\%) were married, $27.30 \%$ were sigle, and the remaining $10.2 \%$ were either divorced or widowed.

Two-tailed $t$-tests were conducted on the demographics across regions. No significant differences were found for respondents' Age, Years on the Job, or Marital Status. The test for Education, however (coded as: 1 = primary; $2=$ secondary; 3 = bachelor degree; and $4=$ masters degree or above), revealed the mean for Zhuhai $(\mathrm{M}=2.32, \mathrm{SD}=0.47)$ to be significantly higher than that for Macau $(\mathrm{M}=2.16, \mathrm{SD}=0.37), p<.01$. Also, Chi-square (Mann-Whitney) tests on the variables of Gender and Industry type showed no significant dissimilarities in the proportions of respondents in these categories across regions.

\section{Materials}

The questionnaire was designed to assess six topic areas, namely, the employee's demographics, YBLI, Optimism, Family Emotional Support, Future Prospects, and Chinese Cultures. The demographics section asked for information about respondents' gender, age, education level, industry in which employed, number of years at current job, marital status, and ethnicity (ethnicity was included to ascertain that the respondents were Chinese).

YBLI. This was measured by the 11 topics developed by OECD (2019), which includes attitude towards housing, education, environment, etc. The reliability for this variable in the current study was .82.

\section{Self-Efficacy}

This was measured using the 23-item Self-efficacy Scale (Sherer et al. 1982). Exmaples of the items include: "I am a self-reliant person" (general self-efficacy) and "when I'm trying to become friends with someone who seems uninterested at first, I don't give up very easily" (social self-efficacy). The scale reliability reached .85 in this study.

\section{Family Emotional Support}

Ten items were selected from Procidano and Heller's (1983) 20-item Perceived Family Social Support scale. A sample item was: "My family gives me the moral support I need." The reliability in this study turned up quite high at .91.

\section{Future Prospects}

The measure of future prospects was adopted from Taormina's (2004) Organizational Socialization Inventory (OSI). Among the four socialization domains: training, understanding, coworker support, and future prospects, only 'future prospects' is selected to test its relationship with YBLI in this study. The domain has five items. One example is: "My company offers good promotion opportunity for me." The reliability score was .88 in this study. 


\section{Chinese Values}

A 12-item scale derived from The Chinese Culture Connection (1987) was used. The questions asked how important some values (listed as adjectives) are to the respondents. The items included: "Perserverance," "thrift," and "having few desires." The reliability in this study turned out to be .83 .

The response format for all the items was a 5-point Likert scale ranging from 1 (strongly disagree) to 5 (strongly agree).

\section{Language of the Questionnaire}

All scales were translated from English into Chinese by a bilingual professor at a university in Macau. Another bilingual professor at the same university examined the translated items for their English meaning and evaluated the two versions as equivalent. Since English is used somewhat in both Zhuhai and Macau, both the English and Chinese phrases were used on the questionnaire to optimize understanding of the items.

\section{Procedure}

Over a period of several weeks, the questionnaires were handed to residents in several large residential areas in Macau and Zhuhai. The residents were approached individually on a random basis, told the general purpose of the study, and asked to complete the questionnaire.

Respondents were assured that their participation was voluntary and that their answers would be kept anonymous. The cover page of the questionnaire agina briefly explained the purpose of this study and guaranteed confidentiality. A total of 446 completed questionnaires ( 231 from Macau, and 215 from Zhuhai) were collected and entered into data analysis.

\section{RESULTS}

\section{Scale Reliability}

The alpha of the five variables, i.e., YBLI, Self-efficacy, Family Emotional Support, Future Prospects, and Chinese Values were $.82, .85, .91, .88$, and .83 , respectively. All were well above .70, indicating a high internal reliability.

\section{Correlations}

To assess the correlations among the main variables, the data for both regions were combined. The data in the analysis included all the five variables. The means, standard deviations, intercorrelations, and scale reliabilities (in brackets) for all the variables are shown in Table 1.

As shown in the table, the correlations between Self-efficacy, Family Emotional Support, Future Prospects, Chinese Values and YBLI were all statistically significant and in the predicted direction.

Table 1. Means, standard deviations, and correlations among YBLI, Self-Efficacy, Family Emotional Support, Future Prospects, and Chinese Values for the Macau and Zhuhai respondents ( $N=446)$.

\begin{tabular}{|l|l|l|l|l|l|l|l|}
\hline \multicolumn{1}{|c|}{ Variables } & \multicolumn{1}{c|}{ Mean } & \multicolumn{1}{c|}{ SD } & \multicolumn{1}{c|}{$\mathbf{1}$} & \multicolumn{1}{c|}{$\mathbf{2}$} & $\mathbf{3}$ & $\mathbf{4}$ & $\mathbf{5}$ \\
\hline 1. YBLI & 3.28 & 1.25 & $(.82)$ & & & & \\
\hline 2. Self-Efficacy & 3.08 & 1.33 & $.31 * * * *$ & $(.85)$ & & & \\
\hline 3. Family Emotional Support & 3.68 & 1.26 & $.45 * * * *$ & $.43 * * * *$ & $(.91)$ & & \\
\hline 4. Future Prospects & 3.39 & 1.32 & $.64 * * * *$ & $.56 * * * *$ & $.42 * * * *$ & $(.88)$ & \\
\hline 5. Chinese Values & 3.16 & 1.43 & $.30 * * * *$ & $.29 * * * *$ & $.59 * * * *$ & $.34 * * * *$ & $(.83)$ \\
\hline
\end{tabular}

${ }^{*} p<.05 ;{ }^{* *} p<.01 ;{ }^{* * *} p<.005 ;{ }^{* * * *} p<.001$ 
Self-efficacy was highly and significantly correlated with YBLI $(r=.31)$, and the same held true for Family Emotional Support ( $r=.45)$, Future Prospects $(r=.64)$, and Chinese Values $(r=.30)$, with all $p$-values $<.001$. These results lent strong support to $\mathbf{H}(\mathbf{1})$ through $\mathbf{H}(\mathbf{4})$.

\section{Tests for YBLI Comparisons}

To test for differences across the Macau and Zhuhai regions, an independent sample, two-tailed t-test was run on the mean of YBLI (with all the 11 topics combined). The test yielded a significant difference, with the Macau mean $(\mathrm{M}=3.30)$ being significantly higher than the Zhuhai mean $(\mathrm{M}=$ $3.25), \mathrm{p}<.005$. This result lent strong support to $\mathbf{H}(\mathbf{5})$. It suggested that Macau residents were more happy and satisfied.

Independent sample, two-tailed t-tests were also run to compare each mean of the 11 topics of YBLI across the two regions. While there was no significant difference for Education, Safety, Civic Environment and Communicty, the Macau means were significantly higher than the Zhuhai means for Life satisfaction, Income, Jobs, Work-life balance and Health. At the same time, the means for Housing and Environment were significantly higher in Zhuhai than those in Macau. The mean scores for comparisons on YBLI and all the 11 topics are shown in Table 2.

\section{Tests for Common-Method Bias and Multicollinearity}

As the data for all the variables were gathered from every respondent, the possibility of commonmethod bias and multicollinearity needed to be ascertained. Common-method bias was assessed by factor analyzing all the variables together, using the maximum-likelihood approach with a forced, one-factor solution. In such an analysis, the resultant Chi-square value is then divided by the degrees of freedom to assess model fit, whereby a ratio of less than 2 to 1 would indicate a good fit (i.e., the

Table 2. Comparison of Macau and Zhuhai mean scores for YBLI and the 11 Topics of YBLI (N = 446)

\begin{tabular}{|c|c|c|c|}
\hline & Macau & Zhuhai & \\
\hline & $(\mathrm{N}=\mathbf{2 3 1})$ & $(\mathrm{N}=215)$ & $t$ \\
\hline YBLI & $3.30(1.06)$ & $3.25(1.45)$ & $1.17 *$ \\
\hline Housing & $3.02(.84)$ & $3.36(1.04)$ & $2.93 * *$ \\
\hline Education & $3.39(.90)$ & $3.35(.92)$ & 0.93 \\
\hline Life Satisfaction & $3.41(1.06)$ & $3.20(1.21)$ & $2.79 * *$ \\
\hline Income & $3.44(1.12)$ & $3.10(1.53)$ & $2.92 * *$ \\
\hline Environment & $3.03(.95)$ & $3.14(.98)$ & $2.35^{* * k}$ \\
\hline Safety & $3.58(1.01)$ & $3.61(.87)$ & 0.70 \\
\hline Jobs & $3.57(1.08)$ & $3.35(1.23)$ & $2.84^{* * *}$ \\
\hline Civic Engagement & $3.48(1.23)$ & $3.45(1.44)$ & 0.93 \\
\hline Work-Life Balance & $2.99(1.25)$ & $2.89(1.32)$ & $2.32 *$ \\
\hline Community & $3.13(.99)$ & $3.10(1.28)$ & 0.71 \\
\hline Health & $3.29(1.34)$ & $3.24(1.44)$ & $1.16^{*}$ \\
\hline Self-Efficacy & $3.09(1.43)$ & $3.07(1.22)$ & 0.70 \\
\hline Family Emotional Support & $3.66(1.03)$ & $3.70(1.49)$ & 0.92 \\
\hline Future Prospects & $3.55(1.54)$ & $3.23(1.09)$ & $2.88 * *$ \\
\hline Chinese Values & $3.01(1.48)$ & $3.32(1.37)$ & $2.80 * *$ \\
\hline
\end{tabular}


presence of common-method bias). For the data in this study, the ratio was 8.87 to1, indicating that common-method bias was not a concern (Podsakoff \& Organ, 1986).

Given the significant correlations among the variables, tests for multicollinearity were run on Optimism, Family Emotional Support, Future Prospects, and Chinese Values as they were to be assessed as possible predictors in the planned regression using YBLI as the criterion variable. The test requires the intended criterion to be excluded while each potential predictor is regressed on the remaining predictors, such that a tolerance $\left(1-R^{2}\right)$ value of less than .10 for any of the regressions would be considered problematic (Winship \& Western, 2016). In the four regressions that were run, the tolerance values ranged from .57 to 1.00 . Since all the values were well above the .10 cutoff, multicollinearity did not appear to be a problem.

\section{Regressions}

Three multivariate regressions were run to determine the effects of Optimism, Family Emotional Support, Future Prospects and Chinese Values on YBLI. One regression used the combined data from both Macau and Zhuhai; while additional, separate regressions were run using only the Macau data, or only the Zhuhai data. In the first regression, for the two regions combined, a total of $50 \%$ of the variance for YBLI was explained, with Future Prospects accounting for most of the explained variance.

In the second analysis, for the Macau sample $(n=231)$, a total of $61 \%$ of the variance was explained, once again with Future Prospects accounting for the majority of the explained variance.

In the separate analysis for the Zhuhai sample $(n=215)$, a total of $40 \%$ of the variance was explained, with both Chinese Values and Self-efficacy accounting for significant amounts of the explained variance. The results of all three regressions are presented in Table 3.

With regard to the power of the findings in the regressions, computations were performed for the combined data of the two regions, and separately for each of the regions. Power was defined using Cohen's (1992) $f^{2}$ "effect" value (where $f^{2}=R^{2} /\left[1-R^{2}\right]$ ), and his cutoff values of .02 for a small effect, .15 for a medium effect, and .35 for a large effect.

For Macau, the total variance explained was $61 \%$, a very large power effect, $f^{2}=1.56$. For Zhuhai, the total variance explained was $40 \%$, representing a large effect, $f^{2}=.67$. When the data for both regions were combined, the total variance explained was $50 \%$, representing a very large effect, $f^{2}=1.00$.

Table 3. Stepwise multiple regressions on YBLI using Self-efficacy, Family emotional support, Future prospects, and Chinese values as predictors for the Macau data $(\mathrm{N}=231)$, Zhuhai data $(\mathrm{N}=215)$, and combined data $(\mathrm{N}=446)$

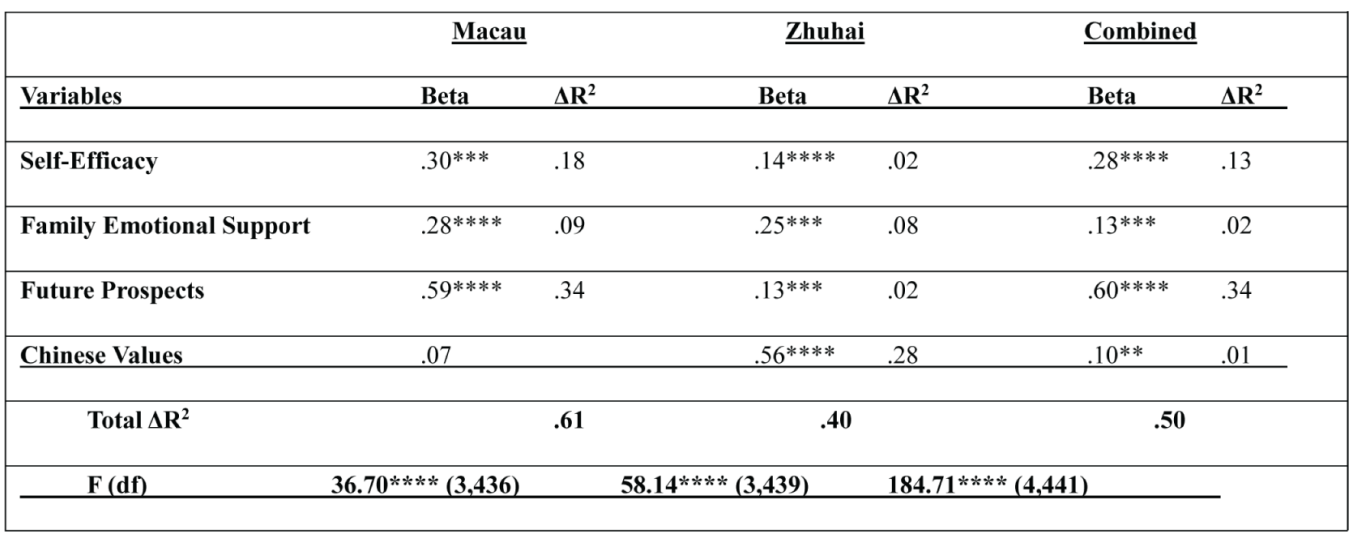

Note: Betas are standardized coefficients. The total df values do not add to N-1 due to listwise deletion of cases with missing data.

$* p<.05 ; * * p<.01 ; * * * p<.005 ; * * * * p<.001$. 


\section{DISCUSSION}

The results revealed that people in the two Chinese regions had very different view on life satisfaction. Moreover, the significant predictors of YBLI in the two regions were also different.

\section{Differences in YBLI}

The mean YBLI score for Macau was significantly higher than that for Zhuhai. This outcome could be due to a stronger economic growth and a better social security system which provides residents with qualified and timely support whenever they need in the Macau SAR, as hypothesized.

Since opening up its locally-controlled casino industry to foreign competition in 2001, Macau has attracted tens of billions of dollars in foreign investment, transforming the territory into one of the world's largest gaming centers. Macau's Gross Domestic Product (GDP) reached 440.3 billion patacas (US\$55 billion) in 2018 and GDP per capita was 666,893 patacas (about US\$82,609). According to the latest report (2018) from the Economist Intelligence Unit (EIU), Macau's economy recorded a growth of $4.7 \%$ in 2018. The 2019 Index of Economic Freedom ranked Macau the $34^{\text {th }}$ freest in the 2019 Index, and the 9th among 43 countries in the Asia-Pacific region with its overall score above the regional and world averages.

Alternatively, it could be argued that there is a higher level of life satisfaction among the Macau residents because of their easy-going, laid back way of living. Given the high mean difference on YBLI between the two regions, the result might also reflect a combined effect of the stronger economy with better social welfare and a more relaxed and causal lifestyle in Macau. Determining which of the factors (or combination of the two) might be responsible for the huge difference could be a topic for future research.

\section{Differences in Future Prospects}

Although no hypotheses were made for differences in the four antecedent variables across regions, the difference in the mean score for Future Prospects in Macau and Zhuhai was quite profound (see Table 2). The Macau mean score was significantly higher than that for Zhuhai. Apparently, the Macau residents see their organizations as offering more opportunities for advancement.

Zhuhai (with a population of 1.89 million people and a geographical territory of 7,649 square kilometers) has had an economic growth focus since it was designated as one of the four earliest Special Economic Zones in China in 1980. By the year 2018, the fixed assets investment there reached US\$185.86 billion (Zhuhai Municipal Government, 2018). Zhuhai has become an attractive place to work for people from all over China because business opportunities are increasing as more enterprises are setting up facilities there. Although Zhuhai is developing fast, with averaged annual growth of more than $9.7 \%$ between 2010 and 2018 (Statistics Bureau of Zhuhai, 2019), Macau's five-year recovery (from 2007 to 2012) laid a solid foundation for its future economic development. A rapid rise in the number of mainland visitors due to China's easing of travel restrictions, increased public works expenditures, and significant investment inflows associated with the liberalisation of Macau's gaming industry enabled it to be one of the wealthiest regions in the world.

Differences in Chinese Values. As discussed before, the two regions have different cultures. Zhuhai has always been a part of mainland China and is guided by the traditionl Chinese Values. Macau, contrarily, follows a Portuguese life style which emphasizes easiness and comfort.

\section{Different Predictors of YBLI Across Regions}

Three regression analyses were run on the data to assess possible predictors of YBLI (see Table 3). To determine the overall effects, one regression was run using the combined data from both regions. In this analysis, as noted earlier, 50\% of the variance for YBLI was explained. Future Prospects accounted for $34 \%$ of the variance, indicating that career advancement opportunity does play a major role in evaluating YBLI. 
When the data were broken down by region, a notable pattern emerged. In Macau, the strongest predictor of YBLI was again Future Prospects, followed by Self-efficacy. Very possibly, the greater opportunities in the Macau economy were reflected in the strong effect that Future Prospects had in predicting YBLI. The presence of Self-efficacy as a predictor might reflect Macau people's confidence in their abilities. In Zhuhai, the two main predictors were Chinese Values and Family Emotional Support. The remaining variance was explained by Future Prospects and Self-efficacy. People in Zhuhai are also quite confident in their abilities and are quite optimistic about their career advancement, however, the values they hold and the support they get from their families affect their ratings of YBLI the most.

\section{Practical Implications}

The correlations and regression results indicated that future prospects and some dimensions (e.g., housing, income, job, etc.) in YBLI are the root causes of disparity. In the world pandemic of 2020, the difference in the two regions is likely to be even more pronounced. Although governments all over the world have implemented economic stimulation packages and income protection schemes (Brewer \& Gardiner, 2020), the Macau government's social assistance measures are multifaceted and powerful. By contrast, mainland residents are more likely to rely on their own savings to tide them over. Policymakers should come up with more citizen-friendly policies to survive the times together.

\section{Theoretical Implications}

The study contributes to the social well-being literature in two ways. First, it exposed that regional (sub) culture makes a large difference in attitudes towards YBLI. In a country as vast as China, subcultures and local perceptions can vary greatly. Second, YBLI is a multidimensional index, affected by personal, organizational, family, and social factors.

\section{Limitations and Implications for Future Research}

The main limitations of this study were the relatively small sample from the two regions (totaling 446 employees), and the number of antecedent variables studied that might influence YBLI. Generally, though, the considerab amount of variance explained for YBLI in the two regions (61\% and 40\%), the high significance level $(p<.001)$ obtained in all the regressions, and the associated large effects (power) of the independent variables suggest that certain domains of personality, organizational factors and social factors significantly impact YBLI. Larger samples should be used in future research to confirm these findings.

A further extension of this model might include more factors that could be tested for their possible influence on YBLI. For example, personality factors, such Openness and agreeableness, should be tested for their relation to YBLI. Similarly, other factors, such as job satisfaction (e.g., with pay, coworkers, and promotions) might also influence YBLI, and should be examined in future studies. Thus, the YBLI variable could offer many opportunities for future research to help clarify the factors that lead residents to be satisfactory toward their life as well as to ascertain the level of quality of their lives.

\section{CONCLUSION}

YBLI is a multidimensional index which reflects people's social well-being and quality of life. This study compared YBLI in two Chinese regions with different sub-cultures. The results revealed that Macau residents were more satisfied than those who lived in Zhuhai, and that predictors of YBLI in the two regions were also different. It implied that policymakers in mainland China need to introduce and implement new policy to improve people's living standards. 


\section{REFERENCES}

Ali, S. R., \& Menke, K. A. (2014). Rural Latino Youth Career Development: An Application of Social Cognitive Career Theory. The Career Development Quarterly, 62(2), 175-186. doi:10.1002/j.2161-0045.2014.00078.x

Botha, F., \& Booysen, F. (2014). Family Functioning and Life Satisfaction and Happiness in South African Households. Social Indicators Research, 119(1), 163-182. doi:10.1007/s11205-013-0485-6

Brewer, M., \& Gardiner, L. (2020). The initial impact of COVID-19 and policy responses on household incomes. Oxford Review of Economic Policy, 36(1), 187-199. doi:10.1093/oxrep/graa024

Cicekli, E., \& Kabasakal, H. (2017). The opportunity model of organizational commitment. International Journal of Manpower, 38(2), 259-273. doi:10.1108/IJM-06-2015-0086

Cohen, J. (1992). A Power Primer. Psychological Bulletin, 112(1), 155-159. doi:10.1037/0033-2909.112.1.155 PMID: 19565683

Coverdale, G. E., \& Long, A. F. (2015). Emotional wellbeing and mental health: An exploration into health promotion in young people and families. Perspectives in Public Health, 135(1), 27-36. doi:10.1177/1757913914558080 PMID:25568200

Deresky, H. (2017). International Management - Managing Across Boarders and Cultures. Pearson.

Direç̧ão dos Serviços de Estatistica e Censos. (2019). Macao Statistics and Census Service.

Garcia, P. R. J. M., Restubog, S. L. D., Bordia, P., Bordia, S., \& Roxas, R. E. O. (2015). Career optimism: The roles of contextual support and career decision-making self-effecicay. Journal of Vocational Behavior, 88, 10-18. doi:10.1016/j.jvb.2015.02.004

Hofstede, G. (2015). Culture's causes: The next challenge. Cross Cultural Management, 22(4), 545-569. doi:10.1108/CCM-03-2015-0040

Horwitz, B. N., Reynolds, C. A., \& Charles, S. T. (2015). Understanding Associations among Family Support, Friend Support, and Psychological Distress. Personal Relationships, 22(1), 79-91. doi:10.1111/pere.12063 PMID:26009699

Index of Economic Freedom. (2019). https://www.heritage.org/index/book/

Jiang, Z. (2016). Emotional intelligence and career decision-making self-efficacy: Mediating roles of goad commitment and professional commitment. Journal of Employment Counseling, 53(1), 30-47. doi:10.1002/joec.12026

Jiang, Z., Hu, X., Wang, Z., \& Jiang, X. (2017). Career decision self-efficacy and life satisfaction in China: An empirical analysis. Social Indicators Research, 132(1), 137-154. doi:10.1007/s11205-015-1201-5

Jin, B., Yu, H., \& Kang, J. H. (2013). Challenges in Western-Chinese business relationships: The Chinese perspective. Marketing Intelligence \& Planning, 31(2), 179-192. doi:10.1108/02634501311312062

Kao, R. H. (2017). The relationship between work characteristics and change-oriented organizational citizenship behavior: A multi-level study on transformational leadership and organizational climate in immigration workers. Personnel Review, 46(8), 1890-1914. doi:10.1108/PR-01-2016-0012

Kwok, S. Y. C. L., Cheng, L., \& Wong, D. F. K. (2015). Family emotional support, positive psychological capital and job satisfaction among Chinese white-collar workers. Journal of Happiness Studies, 16(3), 561-582. doi:10.1007/s10902-014-9522-7

Lelieveld, J., Evans, J. S., Fnais, M., Giannadaki, D., \& Pozzer, A. (2015). The contribution of outdoor air pollution sources to premature mortality on a global scale. Nature, 525(7569), 367-371. doi:10.1038/nature15371 PMID:26381985

Levens, S. M., Elrahal, F., \& Sagui, S. J. (2016). The Role of Family Support and Perceived Stress Reactivity in Predicting Depression in College Freshman. Journal of Social and Clinical Psychology, 35(4), 342-355. doi:10.1521/jscp.2016.35.4.342

Lin, Z., \& Zhao, Z. (2016). Culture, expatriation and performance: Case of Chinese multinational enterprises. Chinese Management Studies, 10(2), 346-364. doi:10.1108/CMS-01-2016-0017 
Minkov, M., \& Hofstede, G. (2011). The evolution of Hofstede's doctrine. Cross Cultural Management, 18(1), 10-20. doi:10.1108/13527601111104269

Nazir, S., Shafi, A., Wang, Q., Nazir, N., \& Tran, Q. D. (2016). Influence of organizational rewards on organizational commitment and turnover intentions. Employee Relations, 38(4), 596-619. doi:10.1108/ER-122014-0150

Pandey, P., \& Asthana, P. K. (2017). An empirical study of factors influencing job satisfaction. Indian Journal of Commerce and Management Studies, 8(3), 96-105.

Peng, Y., \& Mao, C. (2015). The Impact of Person-Job Fit on Job Satisfaction: The Mediator Role of Self Efficacy. Social Indicators Research, 121(3), 805-813. doi:10.1007/s11205-014-0659-x

Podsakoff, P. M., \& Organ, D. W. (1986). Self-reports in organizational research: Problems and prospects. Journal of Management, 12(4), 531-544. doi:10.1177/014920638601200408

Porter, T. H., Kelly, D. R., \& Fields, D. (2016). Work environment and employee motivation to lead. American Journal of Business, 31(2), 66-84. doi:10.1108/AJB-05-2015-0017

Procidano, M. E., \& Heller, K. (1983). Measures of perceived social support from friends and family: Three validation studies. American Journal of Community Psychology, 11(1), 1-23. doi:10.1007/BF00898416 PMID:6837532

Sasson, I. (2016). Trends in Life Expectancy and Lifespan Variation by Educational Attainment: United States, 1990-2010. Demography, 53(2), 269-293. doi:10.1007/s13524-015-0453-7 PMID:26813781

Scheier, M. F., \& Carver, C. S. (1985). Optimism, coping, and health: Assessment and implications of generalized outcome expectancies. Health Psychology, 4(3), 219-247. doi:10.1037/0278-6133.4.3.219 PMID:4029106

Senge, K. (2013). The 'new institutionalism' in organization theory: Bringing society and culture back in. The American Sociologist, 44(1), 76-95. doi:10.1007/s12108-012-9170-5

Taormina, R. J. (2004). Convergent Validation of Two Measures of Organizational Socialization. International Journal of Human Resource Management, 15(1), 76-94. doi:10.1080/0958519032000157357

Tariq, Q. (2012). Self-efficacy and life satisfaction amongst unergraduates. Indian Journal of Positive Psychology, 3(3), 305-308.

The Chinese culture connection. (1987). Chinese values and the search for culture-free dimensions of culture. Journal of Cross-Cultural Psychology, 18(2), 143-164.

Ungerer, L. M., \& Strasheim, A. (2011). The moderating effect of living standards on the relationship between individual-level culture and life satisfaction: A value segmentation perspective. Management Dynamics, 20(3), 25-50. PMID:21941815

Vaupel, J. W., Zhang, Z., \& van Raalte, A. A. (2011). Life expectancy and disparity: An international comparison of life table data. BMJ Open, 1(1), 1-6. doi:10.1136/bmjopen-2011-000128 PMID:22021770

Winship, C., \& Western, B. (2016). Multicollinearity and model misspecification. Sociological Science, 3 , 627-649. doi:10.15195/v3.a27

Zhuhai Municipal Government. (2018). Zhuhai Year Book.

Jennifer H. Gao holds a Ph.D. in Organizational Behavior from the University of Macau, and is an Associate Professor at the School of Business, Macao Polytechnic Institute. She has taught in Ji Nan University in mainland China, Macao University of Science and Technology, and the University of Macau. She has over 10 years of managerial experience in various industries in Macao, Hong Kong, and New Zealand. Her research interests include human resource management, organizational behavior, cross-cultural comparisons, and leadership excellence. She has published in more than 20 international journals, conferences and books. She served on the editorial board of a few books, and now is an ad hoc reviewer for several scholarly journals. 